\title{
Comprensión de gráficas y tablas estadísticas de estudiantes para profesor de educación primaria
}

\section{Comprehension of statistical graphs and tables by primary school teachers-in-training}

\author{
Daniel Eudave Muñoz ${ }^{1}$ \\ Ana Cecilia Macías Esparza ${ }^{2}$ \\ Margarita Carvajal Ciprés ${ }^{3}$ \\ María Guadalupe Muñoz Macías ${ }^{4}$
}

\section{Resumen}

La comprensión de gráficas y tablas estadísticas es fundamental para el desempeño de los profesores de educación primaria, por ser un contenido curricular de la educación básica, y por su utilización en la realización de diagnósticos y planeaciones educativas. Por tanto, la formación inicial de los docentes debe contemplar estos temas. Se reporta una investigación exploratoria-descriptiva realizada en dos fases: en la primera se aplicó una prueba para analizar una gráfica y una tabla estadística, a los 240 alumnos de la Licenciatura en Educación Primaria, en una Escuela Normal, en México; en la segunda, se realizaron entrevistas centradas en tareas a una muestra de nueve alumnos, para reconocer sus comprensiones y dificultades. Los resultados muestran diferentes niveles de comprensión de la gráfica y la tabla, así como de los conceptos estadísticos fundamentales subyacentes. Los hallazgos sugieren que los estudiantes no han trabajado lo suficiente con gráficas y tablas estadísticas.

Palabras clave: Formación inicial de profesores; Comprensión de gráficas y tablas; Sentido estadístico; Ideas estadísticas fundamentales.

\begin{abstract}
Understanding statistical graphs and tables is essential for elementary school teachers' performance, as they are part of the basic education curriculum content; moreover, they are used for the elaboration of educational planning and diagnosis. For this reason, teachers' initial training must consider these subjects. An exploratorydescriptive research, which was made in two stages, is reported: in the first stage, 240 students of the B.A. in Elementary Education at a Teacher Education School in Mexico took a test to analyze a statistical table and a graph; in the second stage, a series of task-focused interviews were applied to a sample of nine students in order to recognize their level of comprehension and difficulties. The results demonstrate different levels of comprehension of the graph and the table, as well as the basic underlying statistical concepts. The findings suggest that students have not worked enough with statistical graphs and tables.
\end{abstract}

\section{Submetido em: 27/09/2019 - Aceito em: 16/01/2020 - Publicado em: 14/02/2020}

${ }^{1}$ Doctor en Educación, profesor-investigador de la Universidad Autónoma de Aguascalientes, México. Email: deudave@correo.uaa.mx.

${ }^{2}$ Doctora en Investigación Educativa, profesora-investigadora de la Universidad Autónoma de Aguascalientes, México. Email: acmacias@hotmail.com.

3 Maestra en Educación, profesora-investigadora de la Universidad Autónoma de Aguascalientes, México. Email: mcarvaja@correo.uaa.mx.

\footnotetext{
${ }^{4}$ Maestra en Ciencias de la Educación. Email: lupi-89@ hotmail.com.
} 
DOI: $10.20396 /$ zet.v28i0.8656854

Keywords: teachers' initial training; graphs and tables comprehension; statistical sense; fundamental statistical ideas.

\section{Antecedentes}

En las últimas décadas la estadística se ha incorporado como un contenido de la educación básica en muchos países, por la necesidad de dotar a todos los ciudadanos de una cultura estadística que les permita comprender la gran cantidad de información presente en múltiples medios impresos y electrónicos, sobre todo tipo de temáticas y con diferentes fines (Batanero, 2002). Esto tiene como consecuencia natural la implementación de estrategias de formación y actualización de los profesores que tienen que atender esta demanda educativa, lo mismo que el desarrollo de múltiples propuestas didácticas para lograr los mejores resultados. En el caso de México, fue en el plan de estudios de 1972 para la educación primaria (edades de 6 a 11 años), que por primera vez se introducen temas de estadística tales como el registro de datos, la noción de frecuencias y las gráficas de barras, así como temas elementales de probabilidad (Ávila, 1998). En las subsecuentes reformas curriculares de la educación primaria, realizadas en 1980, 1993, 2009, 2011 y 2017, se han mantenido los temas de estadística y probabilidad, dándose a partir del plan de 1993, más énfasis al trabajo de obtención y análisis de datos estadísticos y sus representaciones gráficas y tabular. En el ciclo escolar 2019-2020, hay una transición entre el plan de estudios 2011 y 2017, implementándose el primero del tercer al sexto grado y el plan 2017 en primero y segundo grado (SEP, 2019).

En el plan y programas de estudios 2011 de matemáticas para la educación primaria (SEP 2011), se incluye el eje temático Manejo de la Información y el sub-eje Análisis y Representación de Datos, con contenidos a desarrollar a partir del tercer grado y hasta el sexto grado de primaria. En este eje temático se contemplan actividades tales como: representación e interpretación de tablas o pictogramas de datos cuantitativos o cualitativos recolectados en el entorno; lectura de información contenida en gráficas de barras; lectura de información explícita o implícita contenida en distintos portadores dirigidos a un público en particular; resolución de problemas en los cuales es necesario extraer información de tablas o gráficas de barras; identificación y análisis de la utilidad del dato más frecuente de un conjunto de datos (moda); análisis de las convenciones para la construcción de gráficas de barras y el cálculo de la media (promedio) y el análisis de su pertinencia respecto a la moda. Finalmente se incluye la lectura de datos contenidos en tablas y gráficas circulares, para responder a diversos cuestionamientos; el uso de las tres medidas de tendencia central, la media, mediana y moda, en la resolución de problemas. El plan 2017 (SEP, 2017), sigue una lógica similar al plan 2011, dándo énfasis al desarrollo de actividades relacionadas con tareas cotidianas, enfocadas a la obtención de datos, construcción y análisis de tablas y gráficas, así como a las medidas de tendencia central. Aunque hay ligeras variaciones en cuanto al grado escolar en que se presentan algunos contenidos en ambos planes de estudios, el de 2011 y el de 2017, los aprendizajes esperados no varías de manera relevante, estando el énfasis en la 
construcción y lectura de tablas y gráficas sencillas, así como un primer acercamiento a las medidas de tendencia central.

En cuanto a la formación de profesores de primaria en México, ésta se realiza en las Escuelas Normales. Existe un plan de estudios único para la formación de profesores de primaria en todo el país. Al inicio del ciclo escolar 2019-2020 también hay una transición en la Licenciatura en Educación Primaria, entre el plan de estudios 2012 (SEP, 2012 a) y el plan 2018 (SEP, 2018). En México, los estudios previos para obtener el grado de licenciatura son nueve años de educación básica -primaria y secundaria-, y tres años de educación media superior. En ambos planes de estudios se incluyen asignaturas para la formación en didáctica de las matemáticas, enfocadas a la Aritmética (uno en el plan 2012 y dos en el plan 2018), Álgebra, Geometría, y otro más a la Estadística y la Probabilidad (en el plan 2012 con el nombre de Procesamiento de Información Estadística; en el plan 2018 con el nombre Probabilidad y Estadística). Los propósitos, el enfoque y los contenidos en estos dos últimos cursos son muy similares. A continuación, haremos una breve descripción del programa de la asignatura de Procesamiento de Información Estadística, del plan 2012, por ser el que cursaron los estudiantes que participaron en esta investigación. El curso de Procesamiento de Información Estadística se conforma por cuatro unidades temáticas: 1) Estadística Descriptiva (incluye los temas de tablas de distribución de frecuencias y representaciones gráficas; medidas de tendencia central; medidas de posición; medidas de dispersión; estudio de poblaciones con datos vibariados); 2) Probabilidad y muestreo; 3) Inferencia estadística; 4) Vinculación con el eje Manejo de la Información (que incluye los temas de análisis de los conceptos del eje Manejo de la Información y Estadística en la educación primaria, su importancia y retos; desarrollo de estrategias didácticas) (SEP, 2012 b). Esta asignatura, además de ofrecer la formación didáctica, también tiene como propósito lograr que el futuro docente comprenda y aplique los conceptos y procedimientos básicos de la probabilidad y de la estadística descriptiva e inferencial, en la recolección, organización, presentación y análisis de datos para el conocimiento y resolución de problemas en el contexto educativo; de igual forma, se espera que apliquen estos conocimientos en la investigación que tienen que elaborar para obtenter su grado de licenciados. Aunque es evidente el desafece temporal entre el plan de estudios de primaria de 2011 y el plan de la Licenciatura en Educación Primaria implementado desde el 2012, en el caso de los contenidos de estadística no ha habido muchos cambios en los últimos veinticinco años, por lo que consideramos que la formación estadística de los profesores de primaria es congruente con los propósitos del nivel educativo en el que trabajarán.

\section{Las gráficas y tablas como recursos sintéticos de divulgación estadística}

Una competencia básica para lograr una cultura estadística, es la capacidad para leer e interpretar gráficas y tablas estadísticas, ya que estos formatos son recursos privilegiados para agrupar y sintetizar grandes cantidades de información, de manera eficiente y visualmente atractiva. No solo son ampliamente utilizados por los medios masivos de comunicación, son también parte importante de la divulgación de estadísticas oficiales y de los reportes de 
investigación en una gran cantidad de campos del saber (Arteaga, Batanero, Cañadas \& Contreras, 2011; Estrella, 2014). Un ciudadano estadísticamente educado debe tener la capacidad de organizar datos, construir gráficas y tablas y trabajar con diferentes tipos de representaciones de datos, así mismo debe comprender conceptos, vocabulario y los símbolos con que se representan, incluyendo la comprensión de la noción de probabilidad y la medición de la incertidumbre, en suma, debe poseer una alfabetización estadística (Ben-Zvi \& Garfield, 2004, p. 7). En el campo profesional, se requiere también de la capacidad de obtener, procesar y analizar información procedentes de fuentes oficiales, enfrentando a los usuarios a recursos y tareas que pueden ser muy complejos (Gal \& Murray, 2011), como en el caso de los profesores, que enfrentan diferentes necesidades, desde la elaboración de sus registros mensuales o semestrales de calificaciones y demás indicadores del despeño de sus alumnos, y hasta el manejo de las distintas estadísticas que ofrecen organismos oficiales, como la Secretaría de Educación Pública (SEP) y el Instituto Nacional de Estadística y Geografía (INEGI).

Sin embargo, varios estudios realizados con profesores de diferentes niveles educativos y estudiantes para profesor, nos muestran que tienen un desempeño limitado al enfrentar tareas de construcción e interpretación de gráficas y tablas, así como una serie de dificultades que muestran comprensiones incorrectas o poco desarrolladas en cuanto a los criterios básicos para la elaboración y lectura de este tipo de registros estadísticos y de las nociones estadísticas representadas en ellos (Batanero, Arteaga \& Ruiz, 2009; Jacobbe \& Horton, 2010; Juárez \& Inzunsa, 2014; Estrella 2014; Estrella, Olfos \& Mena-Lorca, 2015; Arteaga, Batanero, Contreras \& Cañadas, 2015; Arteaga, Díaz-Levicoy \& Batanero, 2018; Arredondo, García \& López, 2019). Aunque hay algunas dificultades comunes para entender un gráfico o tabla, hay ciertas características propias de cada tipo de registro que conviene analizar por separado.

La complejidad de una gráfica radica en el total de sus elementos, su naturaleza y composición, que podemos sintetizar de la siguiente manera (adaptado de Kosslyn, 1985, citado por Arteaga et al., 2018):

- $\quad$ Estructura: ya sea que tenga forma geométrica (como las gráficas circulares o de sectores, el uso de barras o rectángulos), o se elabore a partir de unos ejes cartesianos.

- Contenido pictórico: por ejemplo, los rectángulos de los histogramas, puntos en los diagramas de puntos y dispersión, líneas, etc.

- Rótulos: encabezados, pie de página, denominación de las variables y los ejes, rango de valores de las variables.

- Contenidos matemáticos: conjuntos numéricos utilizados, las áreas en un gráfico de sectores, etc.

- Contenidos estadísticos: frecuencias absolutas, frecuencias relativas, variación, dispersión, tendencia central, etc. 
Las dificultades en la lectura y en la construcción de gráficas estadísticas tienen que ver con no reconocer todos o algunos de los elementos que las componen y los múltiples significados que encierran (como la naturaleza de los datos que se representan, y las relaciones que se establecen entre ellos). Arteaga el al. (2015) y Arteaga et al. (2018) describen cuatro niveles de complejidad semiótica que se presentan en la construcción de gráficas estadísticas:

N1. Representación de datos individuales. Los alumnos hacen gráficos que representan un solo dato o una porción de los mismos, sin tomar en cuenta la distribución de todos los valores medidos.

N2. Representación de todos los datos individuales de una o varias variables, sin llegar a resumir su distribución. Cuando los alumnos, por ejemplo, representan todos los datos de un conjunto de valores de la estatura de un grupo de personas, pero sin tomar en cuenta el orden de los mismos y, por lo tanto, sin tener clara la noción de frecuencia y distribución.

N3. Representar la distribución de cada variable con gráficas diferentes. En este nivel, los alumnos son capaces de representar los datos a partir del cálculo de las frecuencias, representándolos de forma ordena y dando cuenta de su distribución, pero sin integrar más de una variable en su representación.

N4. Representar varias distribuciones en un mismo gráfico. Los alumnos que tienen este nivel, puede representar las distribuciones de los datos de dos o más variables, como por ejemplo representar en una sola gráfica las frecuencias de la estatura de un grupo de personas, separándolas por su género.

Estos niveles nos muestran la apropiación gradual de los diferentes elementos, códigos y convenciones de representación de las gráficas estadísticas, pero también la apropiación de las nociones que puede generarse a partir de un conjunto de datos, tales como frecuencias, variación, distribución, tendencia central, etc. Estos niveles, como comentaremos más adelante, también explican los procesos de lectura y análisis de gráficas y tablas.

Condiciones similares podemos encontrar con respecto a las tablas estadísticas:

A pesar del papel central de las tablas en la práctica científica, así como su amplia utilización en las clases de ciencias y textos científicos, hay evidencia que la interpretación de las tablas no es una tarea fácil y la adquisición de habilidades de interpretación de tablas no es transparente (Estrella, 2014, p. 16).

Esto se explica en parte por la gran variedad de tipos de tablas, que pueden ir desde listados sencillos de valores correspondientes a una sola variable, hasta complejos arreglos que muestran información conjunta de múltiples variables. De forma genérica, podemos decir que una tabla es:

Un arreglo rectangular con una estructura que comprende un conjunto de filas y columnas, permite presentar los datos correspondientes a una o más variables (características del fenómeno bajo estudio) en forma clasificada y resumida, para permitir la visualización del 
DOI: $10.20396 /$ zet.v28i0.8656854

comportamiento de los datos y facilitar la comprensión de la información que se puede extraer. (Estrella, 2014, p. 6).

En una tabla, por lo general una fila corresponde a una clase de casos y una columna corresponde a una variable. Al igual que las gráficas, en las tablas se tiene una serie de convenciones para su construcción, y aunque pueden variar algunos elementos o criterios, lo más común es identificar los que reseña Estrella (2014): título, cuerpo de datos (bloque rectangular conformado por filas, columnas y celdas que contienen información numérica), encabezado lateral o primera columna, encabezado superior (con el nombre del contenido de las columnas), totales (en la última fila y/o la última columna). También son importantes otros elementos del formato de una tabla: La disposición o ubicación espacial de los elementos; los agrupamientos (tipos de ordenamiento de los datos según algún criterio); el número de cifras de los números representados; el tipo de medidas estadísticas (frecuencias absolutas o relativas, tendencia central, etc.); los códigos no verbales ni numéricos que facilitan la visualización de los elementos de tabla, tales como: forma, tamaño, simbología, color, sombreados, uso de negrita, uso de líneas y espacios, fuentes diferentes, íconos. (Estrella, 2014). Esta autora señala que al igual que las gráficas, las tablas representan una serie de dificultades a los estudiantes, tanto para su construcción como para su lectura, y que dichas dificultades tienen que ver con procesos cognitivos y gráficos, o mejor dicho, semióticos. En estos procesos de comprensión también entran en juego otros procesos que no todos los estudiantes dominan, como el registro y organización de datos, categorización, clasificación y la combinatoria. Sin embargo, a diferencia de las gráficas, las tablas exigen un mayor dominio numérico.

Hay que tomar en cuenta que tanto las gráficas como las tablas estadísticas son al mismo tiempo objetos culturales y objetos semióticos complejos (Arteaga, et al., 2011), constreñidos por una serie de convenciones y criterios técnicos que definen sus elementos y estructura (el significante), con el propósito de comunicar ciertos mensajes (el significado). Los usos requeridos por diferentes grupos profesionales condicionan las características de los sistemas de representación, en este caso de las gráficas y tablas como objetos culturales. Así, por ejemplo, las necesidades escolares son de naturaleza distinta a las requeridas para los sistemas de evaluación y planeación educativa a nivel estatal o nacional, o a las estadísticas del sector salud, aunque sean comunes ciertos principios básicos (Gal \& Murray, 2011). Por otro lado, el carácter complejo de estos objetos semióticos radica en que en una gráfica o una tabla no hay una relación biunívoca entre un significante y un significado, que los elementos que las componen poseen múltiples significados, que conjugados se amplifican y potencian (Batanero, et al., 2009).

Esta investigación tiene como propósito describir los conocimientos que tienen los estudiantes para profesores de educación primaria, para interpretar una gráfica y una tabla estadísticas, poniendo especial interés en sus comprensiones y dificultades. A partir de esto se espera en un futuro, promover acciones curriculares y didácticas que sean de utilidad para la formación inicial de docentes de educación básica. 


\section{Metodología}

El estudio consistió en una investigación exploratoria-descriptiva, para identificar las comprensiones y dificultades que muestran los estudiantes para profesores de primaria al enfrentar tareas de análisis de datos estadísticos presentados mediante una gráfica y una tabla de frecuencias. Se realizaron dos acercamientos metodológicos complementarios: el primero fue la aplicación de una prueba escrita; en segundo lugar, se realizaron entrevistas centradas en tareas. La prueba de conocimientos se aplicó a todos los estudiantes de la Licenciatura en Educación Primaria de una escuela formadora de docentes de la ciudad de Aguascalientes, México. En total participaron 240 estudiantes: 74 de $1^{\circ}$ semestre; 71 de $2^{\circ}$ semestre; 54 de $5^{\circ}$ semestre; 41 de $7^{\circ}$ semestre.

Con la entrevista se profundizó en la comprensión y las dificultades de una muestra de nueve estudiantes. Para estas entrevistas se seleccionaron dos tipos de alumnos: aquellos que mostraron una mayor comprensión de la tarea realizada en la prueba escrita y los que presentaron más dificultades en el desarrollo de las tareas. Se seleccionaron tres alumnos por cada semestre $\left(3^{\circ}, 5^{\circ}\right.$ y $\left.7^{\circ}\right)$, sin considerar a los del primer semestre, pues aún no tenían suficiente contacto con las actividades formativas del plan de estudios. Para la realización de las entrevistas se tomaron como base las mismas preguntas de la prueba escrita, pero con la diferencia de que los entrevistadores podían sugerir algunas actividades adicionales y los estudiantes podían explicar con más detalle cómo habían llegado a sus respuestas. Las entrevistas se realizaron en las instalaciones de la institución, en espacios que fueron facilitados y donde no se tuvo interrupciones (aulas o cubículos de los tutores). Las entrevistas fueron realizadas por tres de los integrantes del equipo de investigación, se grabaron y posteriormente se transcribieron para su análisis. La categorización de las respuestas de cada alumno se realizó al menos por dos de los investigadores participantes, para efecto de su triangulación.

\section{Instrumentos}

Antes de diseñar la prueba de conocimientos, se procedió al análisis de las competencias esperadas en el Plan de Estudios 2012 de la Licenciatura en Educación Primaria (SEP, 2012 a). Con este análisis se detectó la importancia para los futuros profesores, del conocimiento y comprensión de la información estadística presente en gráficas y tablas, así como del tipo de representaciones y de información más pertinente para enfrentar las tareas docentes y de planeación escolar y didáctica. Se diseñó un cuestionario conformado por dos tareas, la primera consistía en una serie de preguntas hechas con base en una gráfica de líneas y la segunda, con base en una tabla de frecuencias. Tanto la gráfica como la tabla se obtuvieron de un informe de estadísticas educativas oficiales del estado de Aguascalientes, elaborado por la dependencia de gobierno encargada de la impartición de la educación básica (IEA, 2012). Las respuestas fueron abiertas, para su análisis se hicieron categorías y se revisaron por tres personas, para clasificar las respuestas. Previo a la aplicación del cuestionario a los estudiantes para profesores, se hizo un pilotaje del 
DOI: $10.20396 /$ zet.v28i0.8656854

instrumento con estudiantes de pedagogía de una universidad pública del estado de Aguascalientes. A partir del pilotaje se hicieron algunas correcciones al instrumento.

La gráfica de datos estadísticos. La gráfica incluye tres variables: alumnos de nuevo ingreso (variable continua: número de alumnos inscritos); nivel escolar (variable dicotómica: primaria y secundaria) y ciclo escolar (variable con 9 categorías: desde el ciclo 2004-2005 hasta el ciclo 2012-2013). (Ver Figura 1).

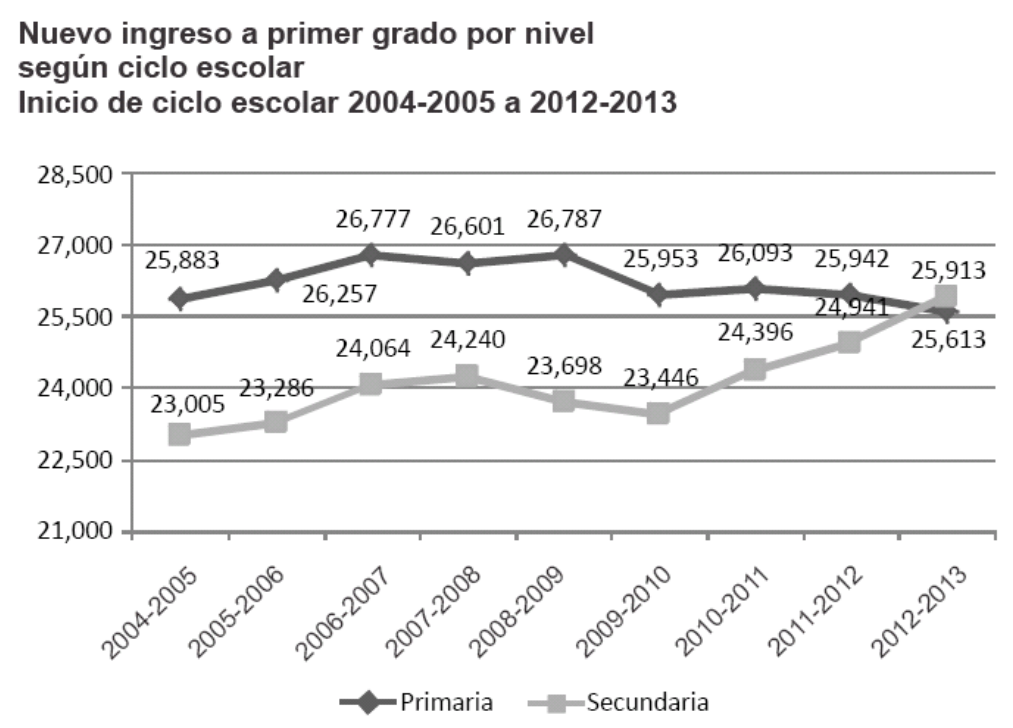

Figura 1. Gráfica presentada en la prueba y en la entrevista.

Fuente: IEA (2012) Las cifras de la educación. Inicio de ciclo 2012-2013. Estadísticas de educación básica.

La gráfica de líneas representa la evolución de la matrícula de la educación primaria y secundaria en el estado de Aguascalientes, México, durante nueve ciclos escolares. Las líneas muestran explícitamente los altibajos en la matrícula de ambos niveles educativos, y las cifras correspondientes a cada año permiten una valoración precisa de dicha evolución. Las cinco preguntas que se presentaron a los estudiantes iban dirigidas a indagar el reconocimiento de la información que presenta la gráfica, de las variables involucradas, del reconocimiento de las tendencias de la variable ingreso a lo largo de los años, tanto para primaria como secundaria, así como el análisis de la variable ingreso año por año (que implica una comparación entre los niveles educativos).

La tabla de frecuencias. En cuanto a la otra tarea, se adaptó una tabla de las estadísticas de educación básica del Estado de Aguascalientes (IEA, 2012), en donde se presentan los datos correspondientes a la inscripción inicial de los alumnos de primaria en el ciclo escolar 2012-2013. En esta tabla, compuesta por 8 columnas y 14 renglones, se incluyen tres variables: grado escolar (de $1^{\circ}$ a $6^{\circ}$ ), edad de los alumnos (de 5 a 15 años), y la inscripción inicial de alumnos (número de alumnos inscritos). Un aspecto que vuelve más 
DOI: $10.20396 /$ zet.v28i0.8656854

compleja la tabla, es la variación que la variable edad muestra con respecto al grado escolar, correspondiendo el valor modal a la edad idónea para cada grado escolar (Ver Figura 2).

Inscripción inicial de alumnos en educación básica primaria Inicio de ciclo escolar 2012-2013

\begin{tabular}{|c|r|r|r|r|r|r|r|}
\hline \multicolumn{7}{|c|}{ Primaria } \\
\hline Edad & \multicolumn{1}{|c|}{$\mathbf{1}^{\circ}$} & \multicolumn{1}{|c|}{$\mathbf{2}^{\circ}$} & \multicolumn{1}{c|}{$\mathbf{3}^{\circ}$} & $\mathbf{4}^{\circ}$ & $\mathbf{5}^{\circ}$ & $\mathbf{6}^{\circ}$ & \multicolumn{1}{c|}{ Total } \\
\hline 5 & 8,106 & 1 & & & & & 8,107 \\
\hline 6 & 17,512 & 7,654 & & & & & 25,166 \\
\hline 7 & 946 & 17,153 & 7,117 & & & & 25,216 \\
\hline 8 & 160 & 1,530 & 17,169 & 7,185 & & & 26,044 \\
\hline 9 & 49 & 285 & 1,791 & 16,534 & 7,122 & & 25,781 \\
\hline 10 & 20 & 89 & 437 & 1,948 & 17,229 & 6,881 & 26,604 \\
\hline 11 & 7 & 29 & 97 & 457 & 2,161 & 16,763 & 19,514 \\
\hline 12 & 2 & 9 & 34 & 120 & 538 & 2,328 & 3,031 \\
\hline 13 & 1 & 2 & 12 & 19 & 99 & 521 & 654 \\
\hline 14 & & 1 & 1 & 3 & 21 & 98 & 124 \\
\hline 15 & & & & 2 & 1 & 12 & 15 \\
\hline & & & & & & & \\
\hline Total & 26,803 & 26,753 & 26,658 & 26,268 & 27,171 & 26,603 & 160,265 \\
\hline
\end{tabular}

Figura 2. Tabla presentada en la prueba y en la entrevista

Fuente: IEA (2012) Las cifras de la educación. Inicio de ciclo 2012-2013. Estadísticas de educación básica.

Para esta tabla, se presentaron tres preguntas, dos orientadas a realizar un análisis comparativo entre la matrícula de los diferentes grados escolares (análisis por columnas para identificar los grados escolares con mayor y menor matrícula), y una destinada a identificar en qué grado escolar se presenta un mayor número de alumnos con extra-edad (con más edad de la ideal para cada grado), lo que implica un análisis de las tendencias de la variable edad en cada grado escolar, y un comparativo entre los diferentes grados.

\section{Resultados}

\section{Respuestas a la prueba escrita}

Si bien alrededor de la mitad de los estudiantes que respondieron la prueba pudieron dar cuenta de la información contenida en la gráfica y en la tabla, era esperable un mejor desempeño, considerando que las actividades de construcción y lectura de este tipo de representaciones se contempla desde la educación primaria y son parte de la formación de los futuros profesores. En general encontramos tendencias de respuestas similares a los resultados reportados por otros autores (Batanero, et al., 2009; Jacobbe \& Horton, 2010; Arteaga et al., 2015; Estrella et al., 2015; Arredondo et al., 2019). 
DOI: $10.20396 /$ zet.v28i0.8656854

Las respuestas a la gráfica estadística. La primera pregunta solicitaba identificar la información contenida en la gráfica (¿Qué información presenta la gráfica?). Tenemos que el $55.4 \%$ lograron identificar los elementos de la gráfica (título, fenómeno educativo que se describe, rasgos sobresalientes), mientras que un 35\% identifica sólo parcialmente el contenido de la tabla. Una porción pequeña $(9.2 \%)$ no reconoce el sentido de la gráfica, a pesar de que esta cuenta con su título y sus elementos son explícitos. Solamente una persona no respondió esta pregunta.

$\mathrm{Al}$ pedirles que identificaran las variables que se describen en la gráfica, únicamente el $12.1 \%$ reconoció las tres variables involucradas (alumnos de nuevo ingreso; nivel escolar; ciclo escolar), lo que implica el reconocimiento de los valores de los fenómenos que se representan en la gráfica. Un $32.9 \%$ reconoce dos variables y un $26.3 \%$ sólo menciona una variable. El $6.3 \%$ menciona otro fenómeno relacionado con el tema de la gráfica, pero sin mencionar ninguna de las variables involucradas. El 17.9\% hizo mención a las diferencias o variaciones entre los datos, pero sin mencionar explícitamente las variables. El $4.6 \%$ no contestó.

Ante la consigna que les solicitaba sus comentarios sobre el nivel de primaria, el $56.7 \%$ pudo hacer una descripción correcta de las tendencias del nuevo ingreso a primaria a lo largo del período considerado, al parecer, apoyándose en la línea que representa esta tendencia (Ver Figura 1), como se pudo corroborar en las entrevistas, lo que implica un análisis visual de los incrementos y decrementos en la matrícula de nuevo ingreso a lo largo del tiempo, pero sin involucrar necesariamente una comparación numérica fina (que sí realizó únicamente un 5.4\%). Es de notar que hubo algunos alumnos que hicieron una descripción incorrecta, aunque fueron pocos (5.8\%). Es interesante que un $27.9 \%$ de los alumnos mencionaran otras cosas asociadas con el fenómeno representado en la tabla, pero que no tenía nada que ver con las variables involucradas en la gráfica, como si el tema de la gráfica fuera un detonante para hacer mención de situaciones asociadas, como el crecimiento poblacional, la construcción de un mayor número de escuelas en la localidad, etc. El 4.2\% no contestó esta pregunta.

De manera similar a lo identificado en la descripción de las tendencias de la matrícula de nuevo ingreso en primaria, para el caso de la matrícula del nivel de secundaria, los porcentajes de respuesta de los alumnos fueron muy similares, predominando una descripción correcta pero sin detalles en el análisis de las cifras correspondientes (59.6\%), esto es, haciendo alusión únicamente a las variaciones que se aprecian directamente en la línea que representa los valores de alumnos de nuevo ingreso, haciendo afirmaciones tales como "se incrementa el número de alumnos", "sube la matrícula a lo largo de los años". Una porción muy pequeña, el 3.8\% hizo una descripción detallada y sustentada en la descripción de las variaciones numéricas. También es similar el porcentaje de alumnos que hacen una descripción incorrecta (5.4\%) o que comenta algo que no tienen que ver con las variables de la gráfica (27.5\%). El 3.8\% no contestó. 
Analizar las tendencias de la matrícula de nuevo ingreso a primaria o a secundaria, por separado, y apoyados en una representación gráfica (una línea en donde es relativamente fácil identificar altibajos) es una tarea que podemos considerar como de nivel 3, según la tipología de Arteaga et al. (2015). Comparar los datos de inscripción de nuevo ingreso a primaria y secundaria a lo largo del tiempo (quinta tarea), implica comparar las dos distribuciones (nivel 4). Comparar la evolución de las cifras de ambos niveles educativos a lo largo del tiempo, identificando numéricamente las variaciones año con año y entre niveles, fue una tarea que pocos alumnos pudieron realizar (9.2\%). Un 22.9\% hizo una comparación de las tendencias entre los dos niveles, pero sin hacer referencia a las cantidades, lo que implica tener una noción correcta del sentido de la gráfica, pero quedarse a un nivel muy intuitivo o visual. Un 38.3\%, aunque pueda comentar algo de las tendencias mostradas por la gráfica, no hace realmente una comparación entre datos. Para un $22.1 \%$, la temática de la gráfica los lleva a comentar otros temas afines, pero no vinculados directamente con la información de la gráfica. El 7.5\%, correspondiente a 18 alumnos, no contestaron.

Podemos suponer que la mayoría de los alumnos encuestados no tienen experiencia en la lectura de gráficas estadísticas (por lo menos del tipo de gráficas que se les presentó), lo que implica que seguramente éste fue un tema poco trabajado en sus estudios de educación básica y media, e incluso en el nivel superior, no obstante ser un tema contemplado en los planes de estudio.

Las respuestas a la tabla estadística. Como lo señalan Estrella (2014) y Estrella et al. (2015), para los alumnos puede resultar más difícil leer una tabla de frecuencias que una gráfica de líneas o de barras, porque implica hacer un comparativo numérico sin ningún tipo de apoyo visual, más aún si la tabla presenta una cantidad considerable de información. Es el caso de la tabla utilizada en esta prueba, pues fue menor el número de alumnos que dieron una respuesta adecuada, incluso en las dos primeras tareas en las que sólo implicaban hacer la comparación entre las cifras del renglón de totales, pero aquí el asunto clave era precisamente saber en qué parte de la tabla encontrar la información requerida.

Únicamente un $15.8 \%$ pudo identificar el grado en donde se dio un mayor incremento en la matrícula (en el quinto grado), y dar un argumento con sustento en los valores de la tabla, para describir o explicar dicho incremento. Un $27.5 \%$ también dio una respuesta correcta, pero no dio una justificación muy consistente o no dio justificación alguna. Es de notar que un poco más de la mitad (52.9\%) contestó sin considerar todas las cifras de la tabla, señalando cualquier otro grado como aquel en el que se dio el mayor incremento de matrícula (haciendo tal vez una comparación únicamente entre dos grados escolares). Un 3.8\% no respondieron. Estas respuestas nos hacen suponer que la mayoría de los alumnos fueron capaces de identificar datos individuales, pero sin reconocer la distribución de los mismos (lo que equivale al nivel 1 de la tipología de Arteaga et al., 2015).

Una situación similar se dio para el caso de la tarea que solicitaba identificar el grado en el que hubo un decremento mayor de alumnos. Esta tarea requería de identificar la mayor diferencia entre dos grados (lo que implica hacer una resta entre los totales de los grados, de 
dos en dos). Entre los grados $1^{\circ}$ y $2^{\circ}, 2^{\circ}$ y $3^{\circ}, 3^{\circ}$ y $4^{\circ}$, y entre $5^{\circ}$ y $6^{\circ}$, se aprecian a simple vista un decremento de la matrícula, pero, ¿entre qué grados hay un mayor decremento? Únicamente un $10.4 \%$ dio una respuesta correcta (entre $5^{\circ}$ y $6^{\circ}$ grado). Un $15.4 \%$ señaló que era el $6^{\circ}$ grado en donde se dio el mayor decremento, pero sin justificar su respuesta a partir de los datos de la tabla, y un 9.6\% sin dar justificación alguna. En esta tarea también un poco más de la mitad (55.8\%) dieron una respuesta sustentada tal vez en un análisis rápido de la tabla (y por tanto incorrecto) o en alguna suposición fundada en su experiencia, pero no en la información que la tabla aporta. Los alumnos que no contestaron esta pregunta fue de $8.8 \%$, el porcentaje mayor de no respuesta de la prueba.

Para responder la tercer y última pregunta relacionada con la tabla de datos estadísticos, consistente en identificar el grado escolar en que hay un mayor número de alumnos con extra edad (con más edad de la esperada para ese grado), habría que determinar primero cuál es la edad ideal correspondiente a cada grado, y a partir de esto, identificar a los alumnos que rebasan dicha edad. Para responder esta pregunta se podían tomar dos criterios. El primer criterio es de tipo pragmático, considerando que los niños ingresan a la primaria a los 6 años, la edad ideal para cada grado sería de 7 para el segundo grado, de 8 para el tercero, y así sucesivamente hasta llegar a los 11 años en sexto grado. El otro criterio es de carácter estadístico y se refleja en la moda de cada grado (que corresponde a 6 años para el primer grado, 7 para el segundo, y así sucesivamente). Si tomamos como punto de referencia cualquiera de los dos criterios anteriores, es en el $6^{\circ}$ grado en donde se aprecia el mayor número de alumnos con extra edad. Un poco más de la mitad señaló que el fenómeno de la extra edad era mayor en el $6^{\circ}$ grado (53.3\%). El 3.8\% de los alumnos encuestados dijo que en el $5^{\circ}$ grado se daba este fenómeno, dando un sustento numérico a su respuesta y un $14.6 \%$ dio la misma respuesta, pero sin dar una razón (el $5^{\circ}$ es el grado escolar en que se aprecia esta situación en segundo lugar, lo que pudo haber favorecido esta respuesta). Un $24.2 \%$ mencionó como respuesta cualquier otro grado escolar y un 4.2 no respondió.

Como veremos a continuación, en las entrevistas los estudiantes presentaron respuestas similares a las que dieron en la prueba, mostrando además otros elementos que permiten apreciar sus comprensiones y dificultades.

\section{Análisis de las entrevistas}

En las entrevistas, a los estudiantes seleccionados se les presentó primero la gráfica, seguida de la tabla. Se les pidió que revisaran cada una de estas representaciones, haciendo una valoración general de las mismas a partir de la pregunta: ¿de qué trata la gráfica o tabla? Para continuar la entrevista, se retomaron las preguntas de la prueba escrita, con la posibilidad de hacer preguntas adicionales para ampliar las respuestas de los estudiantes, esto a criterio de cada uno de los entrevistadores.

El análisis de las respuestas de los alumnos al realizar la tarea de lectura e interpretación de la gráfica y la tabla nos llevó a considerar dos dimensiones paralelas: a) el reconocimiento y comprensión de los elementos de la gráfica y la tabla; b) el reconocimiento 
y comprensión de las variables involucradas y su naturaleza y sus referentes expuestos en los parámetros de la gráfica y la tabla, así como las ideas estadísticas fundamentales de variación y distribución subyacentes en los valores de cada variable. La valoración de la gráfica y la tabla está condicionada en parte por la comprensión que tienen los estudiantes de la variabilidad de los datos estadísticos y los recursos conceptuales que puedan tener para explicarla (como las nociones de frecuencias, valores máximo y mínimo, rango, moda). Pero también tenemos que los conceptos estadísticos, al estar mediadas por las formas de representación, están condicionadas por los recursos que las explicitan (el rango de valores en cada uno de los ejes, la forma de las líneas, para el caso de la gráfica; o en los valores máximo y mínimo, las frecuencias y la moda, expuestos en cada columna, en el caso de la taba).

Apreciamos diferentes niveles de comprensión de los estudiantes entrevistados. En unos casos queda claro su limitado conocimiento de las características de la gráfica y la tabla y de las convenciones para su construcción. Pero en otros casos, también es evidente una idea estática de los fenómenos representados, una especie de negación de todo el rango de valores posibles, aún y cuando están representados en la gráfica y la tabla. Esta invisiblización de los múltiples valores de las variables (sobre todo las variables cuantitativas), nos hace suponer una limitada comprensión de la noción de variación, aunado al desconocimiento de los criterios y convenciones de construcción de este tipo de representaciones. Esto es lo que Arteaga et al. (2015) identifican como representar (o reconocer) sólo resultados individuales, característico del nivel 1 de su tipología. En el caso opuesto, los estudiantes con una mayor comprensión y por tanto con menos dificultades, además de un conocimiento de todos o al menos la mayor parte de los elementos constitutivos de la gráfica y la tabla, demostraron también una mayor comprensión de las nociones de variación y distribución, ya que en sus explicaciones hacían referencia a todos los valores de cada variable e incluso sus tendencias. En estos estudiantes, ¿qué se desarrolló primero, la comprensión de las características de la gráfica y la tabla o las nociones de variación y distribución? Por el tipo de respuestas que brindaron, nos inclinamos por una relación dinámica entre el significante y su significado, que a lo largo del tiempo y con el apoyo de diferentes experiencias, los ha llevado a sus comprensiones actuales.

¿Cómo se manifestó esto en las respuestas de los estudiantes? Primeramente nos llamó la atención el hecho de que los alumnos con menor desempeño iniciaran su análisis haciendo referencia a un aspecto sobresaliente, o mejor dicho, a un elemento que a ellos les pareciera sobresaliente, ya fuera un elemento visual (como por ejemplo la línea superior de la gráfica, que además es la que resalta por su color) o numérico (por ejemplo, la cantidad más grande en la gráfica o en tabla, o un número ubicado en un lugar más visible, como en la fila o columna de totales). Esta pudiera ser una estrategia para iniciar el análisis de una representación, sin embargo, en el caso de los alumnos con menor desempeño, la mayor parte de su análisis se limitó a ese elemento sobresaliente, ignorando o dando menor importante al resto de elementos de la gráfica o tabla $\mathrm{y}$, por tanto, haciendo una lectura incompleta o sesgada. Poner la atención en un elemento sobresaliente y no ver más allá (o hacerlo de 
manera muy limitada) conlleva otro tipo de concepciones, algunas que se hicieron explícitas durante las entrevistas, otras más, implícitas, por ejemplo: dar más importancia a lo pictográfico que a lo numérico; hacer un análisis de datos aislados, sin reconocer la importancia del conjunto de datos representados, y por ende, sus propiedades, entre las que resaltan su variación y su composición como una distribución específica; una comprensión incorrecta o parcial de las nociones estadísticas involucradas, que se refleja con el uso de términos coloquiales, cuando sería esperable la utilización de la terminología especializada (Park, Park, Lee y Lee, 2016, demuestran lo difícil que puede ser llegar a un uso con comprensión de la terminología estadística); y por último, hacer referencia a fenómenos relacionados con el tema de la gráfica y la tabla, pero no representados directamente en las mismas, o lo que es lo mismo, hacer una lectura idiosincrática como la descrita por Arredondo et al. (2019), consistente en opinar con base en la experiencia o las perspectivas personales, sin leer realmente los valores y sus tendencias.

Por el contrario, quienes tuvieron un mejor desempeño, demostraron una comprensión más completa de la tabla: hicieron un reconocimiento de todas las variables representadas en la gráfica y la tabla (denominándolas correctamente según los encabezados de cada representación), el rango de valores de cada variable y sus principales tendencias (ya fuera de cada variable por separado o de manera conjunta); aunque hicieran uso de los recursos pictográficos, su análisis también se sustenta en los valores numéricos; intentaron hacer una lectura a partir de la mayor parte de los números y elementos de la gráfica y la tabla, lo que les permitió identificar la variación en los datos y hacer una descripción de la distribución, aunque fuera aproximada o intuitiva; hay un uso menor de expresiones coloquiales, y por tanto, una mayor apropiación de la terminología especializada y de sus significados; intentan completar su análisis con los elementos de la gráfica o la tabla, sin recurrir a factores externos, y en caso de hacerlo, son fenómenos directamente relacionado con las variables representadas.

Identificamos tres estudiantes con el desempeño más bajo, que pudieran ser ubicados en el nivel 1 de la tipología de Arteaga et al. (2015), y dos que corresponderían al nivel 4. Los cinco restantes pueden ubicarse entre los niveles 2 y 3 (Ver Tabla 1). No está de más aclarar que esta clasificación es aproximada, pues algunos estudiantes mostraron conocimientos que los ubicarían en posiciones intermedias entre los niveles, o bien porque en la gráfica mostraron un mejor desempeño que en la tabla.

Tabla 1. Clasificación de los estudiantes entrevistados según su nivel de desempeño.

\begin{tabular}{|c|l|l|l|}
\hline Semestre & \multicolumn{3}{|c|}{ Estudiantes (con pseudónimo) } \\
\hline $3^{\circ}$ & Carmen. N2 & Irma. N1 & Graciela. N1 \\
\hline $5^{\circ}$ & Gerardo. N3 & Eduardo. N2 & Soledad. N2 \\
\hline $7^{\circ}$ & Arturo. N4 & Eréndira. N3 (N4) & Pilar. N1 \\
\hline
\end{tabular}

Fuente: elaboración propia. 
Resalta el hecho de que los alumnos ubicados en el N1 son en su mayoría del $3^{\circ}$ semestre, y que los estudiantes con un mayor nivel de comprensión son de semestres más avanzado, aunque con algunas excepciones, lo que nos muestra que el progreso en la comprensión de las gráficas y tablas y los conceptos estadísticos involucrados, puede requerir más tiempo del destinado a la asignatura de Procesamiento de Información Estadística, y que el desarrollo de estas comprensiones se alimenta con actividades adicionales, posiblemente desarrolladas en otras asignaturas, como las relacionadas con la evaluación y la planeación educativas.

\section{Conclusiones}

Para los futuros profesores de educación primaria es fundamental tener la capacidad de leer y analizar gráficas y tablas estadísticas. Esta competencia les garantizará un adecuado desempeño al guiar los aprendizajes de sus alumnos, lo mismo que para obtener, comprender y utilizar diferentes fuentes de información de estadísticas oficiales, para fundamentar sus actividades de planeación escolar.

Sin embargo, las limitaciones en la comprensión de la gráfica y la tabla y las dificultades identificadas en al menos la mitad de los estudiantes, nos muestran que la asignatura de Procesamiento de información estadística, se ha quedado corta con el cumplimiento de su objetivo de ofrecer al menos una alfabetización estadística. De igual forma, nos revela que, en otras asignaturas, como en las de Herramientas básicas para la investigación educativa y Evaluación para el aprendizaje, al parecer, se hace un uso limitado de la estadística. Consideramos que el plan de estudios de la Licenciatura en Educación Primaria establece un marco propicio para el desarrollo transversal de la competencia del análisis estadístico, pero para que pueda lograrse, se requiere del trabajo en equipo de los profesores y una revaloración de las prácticas habituales.

Se debe considerar, además, que los antecedentes académicos de los estudiantes para profesor son diversos, lo mismo que sus condiciones y posibilidades culturales y económicas, y que no todos han tenido la oportunidad a lo largo de su formación básica, de enfrentar situaciones en donde la información estadística este presente, como medios impresos, reportes de investigación o acceso a Internet. En estos casos, se requerirá de apoyos especial que ayuden a subsanar estas posibles carencias. El desarrollo de las competencias para interpretar cabalmente gráficas y tablas estadísticas, requiere de mucha práctica y familiaridad con los diferentes tipos de representación, y esto es precisamente una carencia reportada por varios autores (Batanero et al., 2009; Jacobbe \& Horton, 2010; Estrella et al., 2015), por lo tanto, es necesario abrir en el curriculum el mayor número posible de espacios de análisis de este tipo de representaciones, en especial para aquellos estudiantes con más carencias y dificultades.

La insuficiente atención a la formación estadística de profesores de primaria puede tener diversas implicaciones, la más directa y de mayores efectos a largo plazo, tiene que ver con su actividad docente en las aulas. El fomento de una cultura estadística de todas y todos 
DOI: $10.20396 /$ zet.v28i0.8656854

los niños, debe comenzar con la formación de los profesores y con la actualización de los docentes en servicio. Este será un reto más del proceso de transición curricular que se vive en estos momentos en la escuela mexicana y de los que se prevén en el futuro inmediato.

\section{Agradecimientos:}

Esta investigación se realizó con fondos del Programa de Investigaciones Educativas de la Universidad Autónoma de Aguascalientes, México.

\section{Referencias}

Arredondo, E.H., García, J.I. \& López, C. (2019). Niveles de lectura de estudiantes de licenciatura: el caso de una tabla y una gráfica de líneas. Matemática, Educación e Internet, 19 (2)

Arteaga, P., Batanero, C., Cañadas, G. \& Contreras, M. (2011). Las Tablas y Gráficas como Objetos Culturales. Números. Revista de Didáctica de las Matemáticas, 76, 55-67

Arteaga, P., Batanero, C., Contreras, M. \& Cañadas, G. (2015). Statistical Graphs complexity and reading levels: a study with prospective teachers. Statistique et Enseignement, 6 (1), 3 23

Arteaga, P., Díaz-Levicoy, D. \& Batanero, C. (2018). Investigaciones sobre gráficos estadísticos en Educación Primaria: revisión de la literatura. Matemática, Educación e Internet, 18 (1)

Avila, A. (1998). La ensenañza oficial de las matemáticas elementales en México; su psicopedagogía y transformación (1944-1986). México: Universidad Pedagógica Nacional.

Batanero, C. (2002) Los retos de la cultura estadística, Jornadas Interamericanas de Enseñanza de la Estadística, Buenos Aires. Conferencia inaugural.

Batanero, C., Arteaga, P. \& Ruiz, B. (2009). Análisis de la complejidad semiótica de los gráficos producidos por futuros profesores de educación primaria en una tarea de comparación de dos variables estadísticas. Enseñanza de la Ciencia, 28 (1), 141-154

Ben-Zvi, D. \& Garfield, J. (2004) Statistical Literacy, Reasoning, and Thinking: Goals, Definitions, and Challenges. In Ben-Zvi, D., Garfield, J. (Eds.) The Challenge of Developing Statistical Literacy, Reasoning and Thinking (pp. 3-15). Netherlands: Kluwer Academic Publishers

Estrella, S. (2014). El formato tabular: una revisión de literatura. Revista Actualidades Investigativas en Educación, 14 (2)

Estrella, S., Olfos, R. \& Mena-Lorca, A. (2015). El conocimiento pedagógico del contenido de estadística en profesores de primaria. Educação e Pesquisa, 41 (2), 477-493 
DOI: $10.20396 /$ zet.v28i0.8656854

Gal, I. \& Murray, S.T. (2011). Responding to diversity in users' statistical literacy and information needs: Institutional and educational implications. Statistical Journal of the IAOS, 27, 185-195

Instituto de Educación de Aguascalientes (IEA) (2012). Las cifras de la educación. Inicio de ciclo 2012-2013. Estadísticas de educación básica. México: Dirección de Planeación del Instituto de Educación de Aguascalientes

Jacobbe, T. \& Horton, R.M. (2010). Elementary School Teachers' Comprehension of Data Display. Statistics Education Research Journal, 9 (1), 27-45

Juárez, J.A. \& Inzunsa, S. (2014). Comprensión y razonamiento de profesores de Matemáticas de bachillerato sobre conceptos estadísticos básicos. Perfiles Educativos, 36 (146), 14-29

Park, M.S., Park, M., Lee E.J. \& Lee, K.H. (2016). Preservice Teachers' Difficulties with Statistical Writing. In Ben-Zvi, G. y Makar, K. (Eds). The Teaching and Learning of Statistics. International Perspectives (pp. 261-270). Switzerland: Springer.

Secretaría de Educación Pública (SEP) (2011). Plan de estudios 2011. Educación Básica. México: Dirección General de Desarrollo Curricular, Secretaría de Educación Pública.

Secretaría de Educación Pública (SEP) (2012 a). Acuerdo número 649 por el que se establece el Plan de Estudios para la formación de Maestros de Educación Primaria. México: Diario Oficial de la Federación, del 20 de agosto de 2012.

Secretaría de Educación Pública (SEP) (2012 b). Programa del curso: Procesamiento de Información Estadística. México: Secretaría de Educación Pública.

Secretaría de Educación Pública (SEP) (2017). Aprendizajes Claves para la Educación Integral. Plan y programas de estudio para la educación básica. México: Secretaría de Educación Pública.

Secretaría de Educación Pública (SEP) (2018). Acuerdo número 14/07/18 por el que se establecen los planes y programas de estudio de las licenciaturas para la formación de maestros de educación básica. México: Diario Oficial de la Federación, del 24 de julio de 2018.

Secretaría de Educación Pública (SEP) (2019). Hacia una Nueva Escuela Mexicana. Taller de Capacitación. Educación Básica. Ciclo Escolar 2019-2010. México: Secretaría de Educación Pública. 